\title{
Event-based haptic vibration synthesis using a recursive filter for lower limb prosthetics
}

\author{
Pascal Fortin, Martin J.-D. Otis \\ REPARTI Center \\ University of Quebec at Chicoutimi \\ Chicoutimi, Canada \\ Pascal.fortin1@uqac.ca
}

\author{
Vincent Duchaine \\ Laboratoire de commande et de \\ robotique (CoRo) \\ École de technologie supérieure \\ Montreal, Canada
}

\author{
Jeremy R. Cooperstock \\ Centre for Intelligent Machines \\ McGill University \\ Montreal, Canada
}

\begin{abstract}
Providing a safe and rich environment to achieve ambulatory rehabilitation of the elderly, accident victims, or physically impaired patients, has motivated many researchers to develop lower limb prosthetic systems able to transmit physical stimuli at the skin surface. To this end, we propose a novel event-based method of synthesizing the vibratory characteristics of different types of material, such as broken stone, concrete, snow, sand, and earth, during walking, by using automatically generated Infinite Impulse Response (IIR) filters with pseudo-randomized coefficients to ensure a unique vibration at every step. As a first step, theoretical results were obtained by providing a simulated force input signal into the IIR filter's RealTime Simulink model. The outcomes proved to be promising and demonstrated that the synthesized signals are highly comparable to the measured material response in both time and frequency domains. A comparison of the signals obtained by the proposed IIR filter approach and a physical model based technique is presented. Finally, this paper presents a new lower limb prosthetic-skin interface with the capabilities of rendering interactions measured at the foot such as vibrations and pressure points.
\end{abstract}

Keywords- lower limb prosthetic; haptics; vibrotactile; walking, rehabilitation

\section{INTRODUCTION}

In order to fully appreciate the importance of prosthetics technology, one must note that there are nearly 2 million people living with limb loss, and that approximately 185,000 amputations occur each year in the United States alone [1]. A previous study of a sample of prosthetic users revealed that $57 \%$ of the study's population were unsatisfied with their prosthetic devices [2]. Furthermore, the lack of sensory feedback has been identified as one of the main causes of device abandonment [3].

To address these issues, we propose a new automated Infinite Impulse Response (IIR) filter design to synthesize the vibrations that occur during impact between a sound lower limb and various materials. A novel actuated prosthetic-skin interface able to render these impact vibrations and pressure points under the foot is also presented as part of the solution to this problem.

Following a review of the state of the art in technologies for walking rehabilitation, we describe the primary contribution of this paper, a design for measuring and rendering vibrations to a user of a lower limb prosthetic, such that patients can experience a far more engaging interaction with the ground surfaces on which they are walking. We then describe the method used to extract the material's response model, the design and implementation of synthesized vibrations using the IIR filter, and compare the, acceleration signals produced by the system to measurements from a physical model approach proposed by Turchet et al. [4].

\section{RELATED WORKS}

First, a brief overview of the effect of material type on walking parameters is presented. Then, lower limb prosthetics and locomotion interfaces are covered. Finally, the three main vibration synthesis techniques are compared.

\section{A. Walking rehabilitation}

\section{1) Impact of material type on gait parameters}

Marigold and Patla studied the impact of material compliance on the nervous system, and more specifically, how it modifies the vertical center of mass (COM) [5]. They observed that when the patient is walking on compliant material, the COM trajectory changes and its vertical peak decreases in amplitude. This variation is caused by the limbknee flexion after the toe-off stage, which increases in order to compensate for toe penetration in the soil. Cham and Redfern focused on the changes in gait parameters when the walker is anticipating a slippery floor. Their experiments indicated that typical pedestrians can significantly increase the friction coefficient through biomechanical actions [6]. Their results also suggest that the hips and knee muscles are more involved than the ankles to control slip risks. These results were confirmed by Cappellini et al., who demonstrated that when subjects walk upon slippery materials, they tend to adapt their gait parameters to locate their COM over their supporting limb [7]. These experiments employed basic hardware, often consisting of physical lubricants (oil, water, etc.) on different types of floor (vinyl, plywood, etc.). However, greater flexibility and control, and simplified experimental procedures, could be obtained through the use of a virtual environment equipped with haptic feedback devices.

\section{2) Lower limb prosthetics and locomotion interfaces}

Lower limbs prosthetics is a general term. Since amputation impacts on every aspects of a patient's life [8], a great deal of attention is placed on providing the patient with a highly functional prosthesis, regardless of the exact position of amputation. Technological advances have led to the development of powered prostheses, which are designed to act as a sound limb and decrease adaptation time of the patient. 
Active prostheses are controlled by various methods to ensure volitional control by voice [9] and myoelectric signals [10] among others. Although means of controlling active prosthesis are becoming more sophisticated, patients nevertheless lack active feedback from the materials on which they are walking.

In response to this shortcoming, many locomotion interfaces have been developed to simulate different walking surfaces. One of these, the HapticWalker, is a complete robotic virtual environment walking apparatus featuring three degrees of freedom (DOF) at each foot, designed to accommodate the motion of the patient during all of the walking phases [11]. A second interface takes a different approach, driving independent 6 DOF platforms under each foot using an 18cable transmission system [12]. Both of the aforementioned interfaces are limited to simulating relatively large elements such as slopes and stairs and are similarly limited as the prostheses in their inability to render textural or vibration characteristics of the ground surface. A rigid, flat vibrotactile tile featuring a voice-coil actuator was designed to solve this issue. Incorporated into a complete floor [13], these tiles were used to simulate different types of soil [14] and implement a variable-friction coefficient floor.

\section{B. Vibration Synthesis Techniques}

\section{1) Playback approach}

In the domain of vibration synthesis, a commonly used method in the video game and animation industry consists of measuring the vibrations on different types of materials and playing back the recorded signals to the user [15]. This approach benefits from its minimal computational requirements. However, the number of signals per material type is usually limited to a few samples, which overlap between various recordings. This results in the potential for ambiguity and repetition of playback signals, especially during prolonged use [16], which may detract from the patient's sense of immersion, and is thus not suitable for our objectives.

\section{2) Material synthesis approach}

A second approach to vibration and texture synthesis is based on physical modeling. The physical models employed synthesize sounds and/or vibrations by reproducing the results from the interactions between small individual particles composing the materials. These interactions may include impacts, friction or fractures events. Turchet et al. presented such an approach based on Hunt-Crossley interaction [4] in conjunction with a pair of augmented sandals, and demonstrated that users are able to recognize most of the simulated material types [17]. Another physical model approach uses acceleration data, measured during walking on fragile structures such as aluminum foil and paper, to design a spectrum-based synthesis model [18]. The response of the vibrotactile platform itself is compensated by subtracting it from the signal. The obtained signals proved to be highly similar to their measured counterparts, but the platform requires that users have their legs held in an apparatus, ensuring orthogonal contact with the surface.

Although they provide satisfying results, these physical model techniques impose considerable computational requirements in order to operate in real time. Furthermore, the mechanical constraints of the proposed hardware limits their rehabilitation and prosthetic applications. In contrast, we believe that patients must be able to use their own footwear and maintain their liberty of movement throughout rehabilitation exercises.

\section{3) Cascade filters approach}

The alternative approach proposed in this article is based on discrete filters. Previous research using these methods are mostly focused on texture rendering of different materials. Guruswamy et al. propose the design of discrete Infinite Impulse Response (IIR) filters using a time domain version of Prony's method. The impulse response of the filter is fitted to the acceleration signal. When the system is used to synthesize acceleration for a given type of material, impulses of varying magnitudes are fed to the filter to fit the measured particle repartition [19]. Kuchenbecker investigated the rendering of high frequency vibration and textures in the context of teleoperation, applied to the fingers and hands [20] [21]. The employed method consists of measuring acceleration data from a given material at a given applied pressure using a pen-like probe and compensating for grip pressure [22]. Linear predictive coding (LPC) is then used to develop a discrete transfer function corresponding to the measured response under specific surface-probe parameters. This predictive filter is then inverted to be used to produce unique acceleration signals of arbitrary length by placing it in a feedback loop. To achieve this pseudo-randomized signal, the system generates its response based on the scanning speed and a white noise input [16].

\section{PROPOSED SYSTEM}

The filter approach described above seems to be the most appropriate for our application. Following this direction, we propose an event-based technique using IIR filters to generate real-time reproduction of a material's response from the measured force applied by the user during walking. In contrast with previous research focusing on texture, we investigate the vibrations related to an impact between diverse materials and a sound limb. The filter is designed by fitting the previously measured acceleration data acquired on different materials in the frequency domain using an algorithm based on the damped Gauss-Newton method for iterative search [23]. Furthermore, a variation in the values of filter coefficients is applied to ensure that the haptic responses are unique at every step.

During operation, the filter is provided with readings from the force sensors in order to generate the appropriate vibration signals in response to the specific foot-ground interaction. These vibrations are then rendered through the proposed hardware, a novel interface for lower limb prosthetics with the ability to render vibrations and pressure points is presented. It is hypothesized that this device could replicate the perception of geometry under the foot and consequently increase the ability of a patient to balance. This interface represents the main contribution of this paper.

The remainder of this section briefly describes the hardware and methodology that were used to acquire the measurements associated with each type of material. It also describes the vibrotactile apparatus, equipped with resistive force sensors, used to validate our design, as well as the newly proposed prosthetic-skin interface for rehabilitation uses. 


\section{A. Acceleration acquisition device and procedures}

Measurements of different materials are acquired using our instrumented shoe [24]. The analog signal is read by a Microchip PIC24 microcontroller at a fixed sampling frequency of $1 \mathrm{kHz}$.

For each of the material types we investigate, namely, broken stones, concrete, sand, earth and snow, a sequence of 13 heel strikes was performed, resulting in 65 measurements. Acquisition of heel strike data was preferred over the complete step because of the position of the accelerometer and the ease of associating the resulting data with an impulse signal.

The data are processed to design the appropriate filter to fit the given material's impulse response.

\section{B. Vibrotactile feedback}

To verify the feasibility of our approach through rapid prototyping, we validated the effectiveness of our filter design using a vibrotactile tile consisting of a rigid composite platform mechanically optimized for rigidity [14]. The active element is a TST239 Silver voice-coil actuator manufactured by Clark Synthesis. The signal is outputted through a standard consumer grade computer audio interface and amplified using a 50 Watt Class D audio amplifier by Sure electronics. Results of this testing are described in Section V.

For the purpose of rehabilitation using a lower limb prosthesis, we propose an innovative interface to reproduce for the user the tactile sensations of the material's vibration and pressure points. The system, shown in Fig. 1, is composed of a flexible plastic piece held in place using an elastic fabric. Three servomotors and four piezo-ceramic discs are fixed to the plastic surface. Force measurements are acquired under the foot of the prosthetic limb and reproduced using the servos. These signals are also used as input to the filter system described in this article and fed to the piezo-ceramic actuator to render the material vibratory characteristics.

This apparatus was inspired by a previously designed sensory feedback system for hand prostheses [25]. However, the system proposed here is the first one equipped with an insole for sensing interaction with the material and an actuated device able to replicate those interactions at the prosthetic-skin junction.

a)

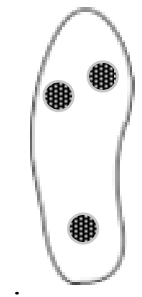

b)

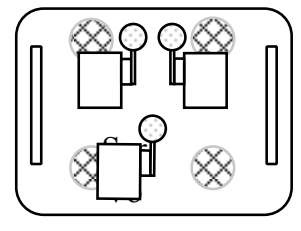

Fig 1. Proposed interface to reproduce tactile sensation of the material's vibration and pressure points in the context of rehabilitation for patients using lower limbs prostheses. (a) insole equipped with force sensors. (b) actuated prosthetic-skin interface. Note that the interface's active components position may vary depending on the patient's limb physical characteristics.

\section{MATERIAL RESPONSE MODEL}

\section{A. Preprocessing}

The next step after acquiring the acceleration signals is the extraction of the material's response. First, the signal has to be parsed manually into a single sequence of heel strikes. The raw acceleration at the heel presented in Fig. 2 contains three main components: high frequency noise introduced by the ADXL335, low frequency trend resulting from the movement of the foot at every impact, and the response of the ground surface, which is the signal of interest.

In order to extract the signal of interest, the two other components must be attenuated. The first step is removing the noise. A $17^{\text {th }}$ order Savitzky-Golay polynomial smoothing filter is used to denoise the signal. To remove the trend caused by the foot's movement, a classic Butterworth band pass filter is used to pass frequencies between 35 and $120 \mathrm{~Hz}$, suitable for the materials covered in this paper. Fig 2 presents an example of the filtering result. Since the audio signal is not investigated here, an analysis of the higher frequency components is irrelevant.
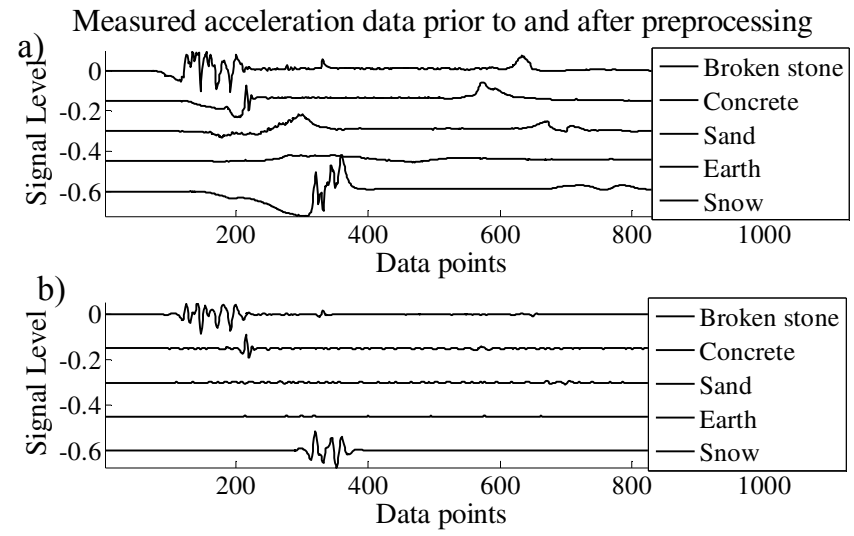

Fig 2. Acceleration data from footsteps. (a) Raw acceleration signal for a single heel strike on each type of ground. (b) Resulting signal after applying a Savitzky-Golay and band pass Butterworth filter.

\section{B. Filter Design}

Once the response from a given type of material is acquired, its discrete Fourier transform is computed. In order to synthesize the acceleration signal, an arbitrary magnitude filter was designed to replicate precisely the frequency response of the system. This filter has the form of

$$
F(z)=\frac{a_{1} z^{-1}+\ldots+a_{M-1} z^{M-1}+a_{M} z^{M}}{b_{0}+b_{1} z^{-1}+\ldots+b_{N-1} z^{N-1}+b_{N} z^{N}}
$$

The set of coefficients is unique for each type of material and is determined by the following algorithm, based on the damped Gauss-Newton method for iterative search by Dennis and Schnabel [26]:

$$
\min _{b, a} \sum_{k=1}^{n} \omega t(k)\left|h(k)-\frac{B(\omega(k))}{A(\omega(k))}\right|^{2}
$$

Where $\mathrm{B}(\mathrm{w}(\mathrm{k}))$ and $\mathrm{A}(\mathrm{w}(\mathrm{k}))$ are the Fourier transforms of polynomials a and $\mathrm{b}$, respectively, at a given frequency vector 
$\mathrm{w}(\mathrm{k})$ of length $\mathrm{n}$. This uses as its initial guess the final results from the algorithm presented by Levi [23].

The order of the filter polynomial coefficients is a crucial factor in precisely reproducing the peaks present in the material response. A too low order filter would not provide the same richness of vibration while a filter whose order is too high requires significantly more resources for real time implementation. Consequently, the order has been determined using an automated iterative process aimed at maximizing the normalized root-mean square error (NRMSE) cost function $\mathrm{C}\left(\mathrm{A}_{\mathrm{S}}(\mathrm{z})\right.$ ) defined in Equation (3) between the synthesized frequency $A_{S}(z)$ and measured frequency response $A_{M}(z)$ while minimizing the order of the system.

$$
C\left(A_{S}(z)\right)=1-\left\|\frac{A_{M}(z)-A_{S}(z)}{A_{M}(z)-\operatorname{mean}\left(A_{M}\right)}\right\|
$$

Furthermore, zero-mean noise with standard deviation of $1 \%$ of the coefficient value is generated and added to each numerator coefficient at every time step. By modifying only the numerator coefficients, as seen in Equation (4), the original stability is maintained, but the position of the zeroes in the zplane causes a variation in magnitude and phase, thereby providing the user with a unique tactile sensation at every footstep. The filter's topology is shown in Fig. 3.

$$
F(z)=\sum_{i=1}^{N} \frac{(1+0.01 \cdot \text { randn }) b_{i} z^{-i}}{a_{i} z^{-i}}
$$

\section{Synthesizing acceleration}

The next step is finding a discrete time transfer function to synthesize the acceleration signal associated with the ground response for a given force input. The said transfer function will be of the form proposed in Equation (4) and is the same as the designed filter.

Experimental results indicate that this design technique creates arbitrary peaks in the $250-500 \mathrm{~Hz}$ range. Therefore, a low pass filter is added to the designed arbitrary magnitude IIR filter to attenuate such undesired peaks in order to comply with the measured signals as shown in Fig. 4.

\section{RESULTS}

The following section presents the results obtained by using the designed filter system in simulation context. At first, its impulse response is compared to the measured acceleration signals. Then, a brief comparison is made between the results obtained with a physical model [4] and the synthesized acceleration signals obtained using our system.

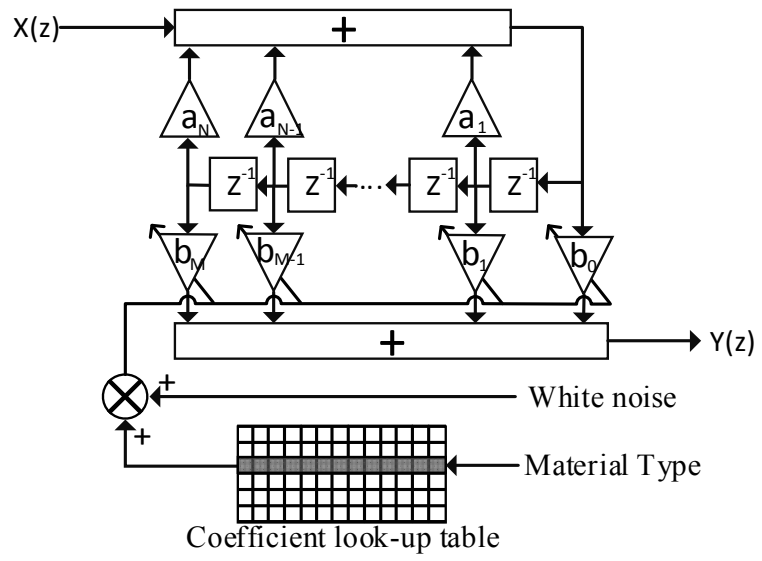

Fig 3. Representation of the filter including the soil type coefficient look-up table and the variable filter coefficients.

\section{A. IIR Filter synthesized signal and measured data}

The ability of the system to synthesize a desired signal depends on how closely the frequency spectrum of the produced signal is matched to the measured data. With this in mind, Fig. 4 presents an example of fitness between the measured acceleration and synthesized signals when excited with a unit impulse for the materials covered in this paper. One can notice from this figure that the fitting algorithm worked flawlessly with most aggregate materials, especially broken stone, sand and earth. The snow signal presents differences but the overall shape of the spectrum is identical. However, the concrete acceleration signal seems to present important oscillations. The effects of such errors will have to be determined through extensive experimentations in order to verify its impact on the perception of the user.

\section{B. Synthesis technique comparison}

Since previous research has addressed vibration synthesis, we are interested in comparing the designed filter system with a physical-model-based approach proposed by Turchet et al. [4].

Their proposed sound synthesis technique is based on the Sound Design Toolkit (SDT) extension to MAX or Pure Data (PD). For the purpose of this article, its PD version was implemented (V.0.9-062, 2013-02-19) using PD-extended (V.0.42.5). The performed tests required us to adjust the impact parameters from the provided "impact-help" files manually. The physical model implemented for impact situations consists of a hammer, hitting a defined material under selected interaction parameters. The user can modify various model variables such as hammer mass, applied force, initial speed, interaction surface, dispersion coefficient, and decay factor. In order to achieve this task efficiently, the measured vibration was played back on the vibrotactile tile followed by the simulation result. Modifications were then applied to appropriate impact parameters to achieve the best possible fit based on the received feedback.

From the simulation results for broken stone and concrete, presented in Fig. 4, the two synthesis methods provide closely related results. However, considering all of the ground types used, the IIR filter approach proves to be superior in terms of 
curve fitting. This is also apparent from the results of Table 1, with lower NRMSE measures for the filter based approach, indicative of a better fit to the modeled data. The shift in frequency spectrum of the physical model approach has a great impact on NRMSE values. Also note that this table only serves to validate the fitness of the designated filter. During experimentation, the coefficient modifications discussed in Section IV B causes variations in NRMSE while remaining well correlated with the original signal.

This comparison of results in itself demonstrates the effectiveness of the IIR filter approach. However, it is also important to note that the two methods are extremely different in terms of implementation. As mentioned previously, in order to implement the different types of material using Pure Data and SDT, the user had to manually set all of the physical model parameters using an iterative process for each type of ground. Having the capacity to add new vibrations is of prime importance. In this regard, the proposed IIR filter design method provides a fully automated process. In order to increase the number of supported types of material, one simply has to input the acceleration measurements to the design algorithm.

TABLE I. NRMSE BETWEEN SYNTHESIZED AND MEASURED SIGNALS

\begin{tabular}{|l|l|l|c|c|c|}
\hline \multirow{2}{*}{$\begin{array}{l}\text { Synth. } \\
\text { method }\end{array}$} & \multicolumn{5}{|c|}{ Normalized Root Mean Square Error } \\
\cline { 2 - 6 } & Broken stone & Concrete & Sand & Earth & Snow \\
\hline $\begin{array}{l}\text { IIR } \\
\text { Filter }\end{array}$ & 0.0287 & 0.1052 & 0.0817 & 0.0715 & 0.0734 \\
\hline $\begin{array}{l}\text { Phys. } \\
\text { Model }\end{array}$ & 3.2380 & 2.3312 & 0.1689 & 35.444 & 1.8997 \\
\hline
\end{tabular}

\section{CONCLUSION AND FUTURE WORK}

A novel event-based IIR filter approach to vibration synthesis has been designed and compared to a physical model approach. The simulation results prove that the proposed filter method provides similar and superior results when compared to Pure Data SDT impact tools. The advantages of the proposed design technique stem from the fact that it can automatically generate the filter coefficients from a measured acceleration signal, thus reducing the time required to add the response of new materials to the system. Furthermore, the pseudorandomly modified coefficients of our approach provide unique vibrations at every step, which we believe is key to maintaining the patient's sense of immersion.

Performance tests should be attempted with human patients, comparing both synthesis methods. Also, the proposed interface aimed at the rehabilitation of patients using lower limb prostheses seems very promising and will be fully implemented in future work.
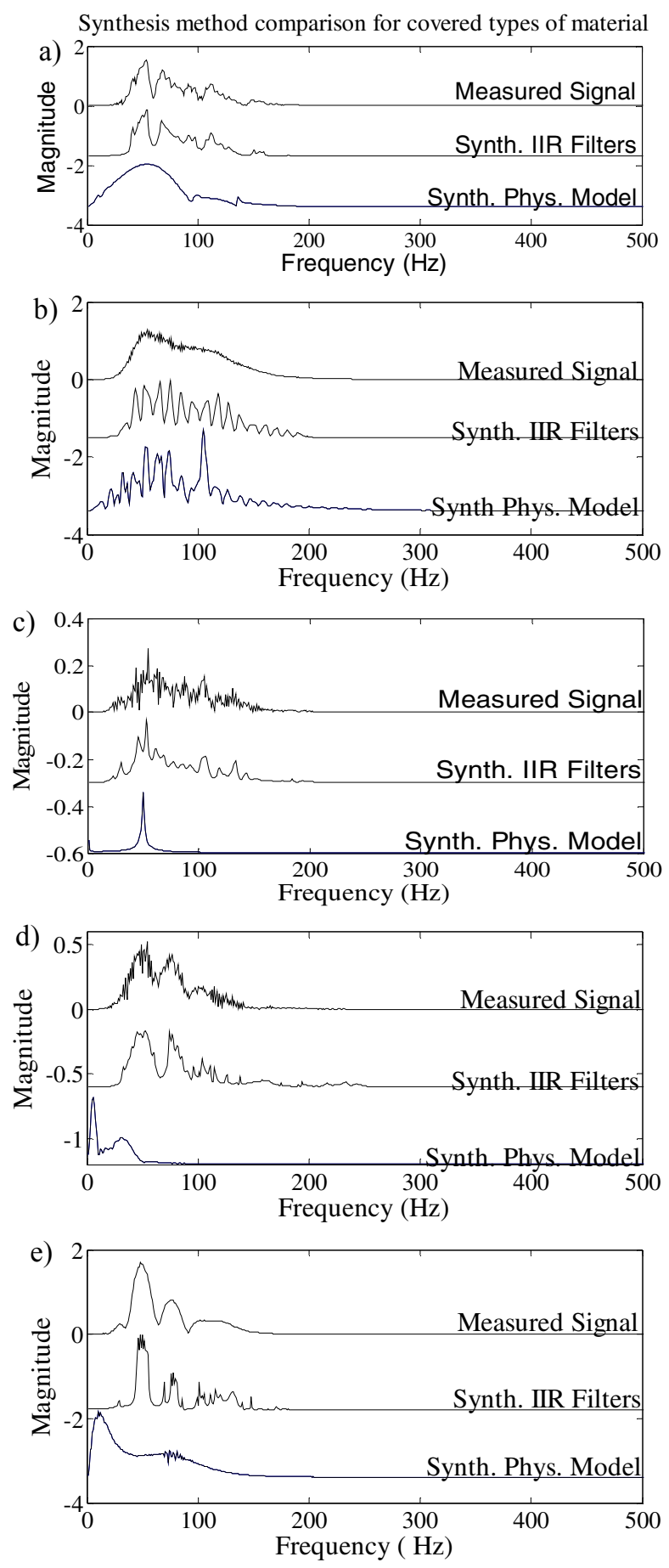

Fig 4. Comparison of the frequency spectrum of the measured signal, IIR filter synthesized signal, and physical model synthesized signal. From top to bottom: (a) broken stone, (b) concrete, (c) sand, (d) earth, and (e) snow.

\section{REFERENCES}

[1] K. Ziegler-Graham, E. J. MacKenzie, P. L. Ephraim, T. G. Travison, and R. Brookmeyer, "Estimating the prevalence of limb loss in the United States: 2005 to 2050," Archives of physical medicine and rehabilitation, vol. 89 , pp. 422-429, 2008 
[2] T. R. Dillingham, L. E. Pezzin, E. J. MacKenzie, and A. R. Burgess, "Use and satisfaction with prosthetic devices among persons with trauma-related amputations: a long-term outcome study," American Journal of Physical Medicine \& Rehabilitation, vol. 80, pp. 563-571, 2001.

[3] E. Biddiss, D. Beaton, and T. Chau, "Consumer design priorities for upper limb prosthetics," Disability \& Rehabilitation: Assistive Technology, vol. 2, pp. 346-357, 2007.

[4] L. Turchet, R. Nordahl, S. Serafin, A. Berrezag, S. Dimitrov, and V. Hayward, "Audio-haptic physically-based simulation of walking on different grounds," in 2010 IEEE International Workshop on Multimedia Signal Processing (MMSP), 2010, pp. 269-273.

[5] D. S. Marigold and A. E. Patla, "Adapting locomotion to different surface compliances: neuromuscular responses and changes in movement dynamics," Journal of neurophysiology, vol. 94, pp. 17331750,2005 .

[6] R. Cham and M. S. Redfern, "Changes in gait when anticipating slippery floors," Gait \& Posture, vol. 15, pp. 159-171, 2002.

[7] G. Cappellini, Y. P. Ivanenko, N. Dominici, R. E. Poppele, and F. Lacquaniti, "Motor patterns during walking on a slippery walkway," Journal of neurophysiology, vol. 103, pp. 746-760, 2010.

[8] J. Abed Saeedi and A. Darvish Pourkhaki, "Experience of Patients with Lower Limb Amputation," Journal of Qualitative Research in Health Sciences, vol. 3, pp. 93-103, 2014.

[9] D. Priyadharshini and R. Subhasree, "Autonomous Control of Lower Limb with Prosthetic Part for Paraplegic Individuals through Voice," Automation and Autonomous System, vol. 5, pp. 211-214, 2013.

[10] O. Mazumder, A. S. Kundu, and S. Bhaumik, "Generating gait pattern of myoelectric active ankle prosthesis," in Recent Advances in Engineering and Computational Sciences (RAECS), 2014, pp. 1-6.

[11] H. Schmidt, "HapticWalker-A novel haptic device for walking simulation," in Proc. of'EuroHaptics, 2004.

[12] M. J.-D. Otis, D. Laurendeau, and C. Gosselin, "Reduced-scale cabledriven locomotion interface for rehabilitation and training," in Canadian Medical and Biological Engineering Conference (CMBEC34), Toronto, Canada, 2011, p. 4.

[13] M. J.-D. O. Guillaume Millet, Gary Chaw, Jeremy R. Cooperstock, "Initial development of a variable-friction floor surface," in Canadian Medical and Biological Engineering Conference (CMBEC'34), FICCDAT, Toronto, Canada, 2011.

[14] Y. Visell and J. R. Cooperstock, "Design of a vibrotactile display via a rigid surface," in IEEE Haptics Symposium, 2010, pp. 133-140.
[15] K. Collins, Game sound: an introduction to the history, theory, and practice of video game music and sound design: Mit Press, 2008.

[16] J. M. Romano, T. Yoshioka, and K. J. Kuchenbecker, "Automatic filter design for synthesis of haptic textures from recorded acceleration data," in IEEE International Conference on Robotics and Automation (ICRA), 2010, pp. 1815-1821.

[17] R. Nordahl, A. Berrezag, S. Dimitrov, L. Turchet, V. Hayward, and S. Serafin, "Preliminary experiment combining virtual reality haptic shoes and audio synthesis," in Haptics: Generating and Perceiving Tangible Sensations, ed: Springer, 2010, pp. 123-129.

[18] S. Okamoto, S. Ishikawa, H. Nagano, and Y. Yamada, "Spectrum-based vibrotactile footstep-display for crinkle of fragile structures," in IEEE International Conference on Robotics and Biomimetics (ROBIO), 2011, pp. 2459-2464.

[19] V. L. Guruswamy, J. Lang, and W.-S. Lee, "IIR Filter Models of Haptic Vibration Textures," IEEE Transactions on Instrumentation and Measurement, vol. 60, pp. 93-103, 2011.

[20] K. J. Kuchenbecker and G. Niemeyer, "Improving Telerobotic Touch Via High-Frequency Acceleration Matching," in IEEE Int. Conference on Robotics and Automation, Orlando, FL, 2006, pp. 3893-3898.

[21] K. J. Kuchenbecker, "Improving Contact Realism through Event-Based Haptic Feedback," IEEE Transactions on Visualization and Computer Graphics, vol. 12, pp. 219-230, 03/01 2006.

[22] J. Fiene, K. J. Kuchenbecker, and G. Niemeyer, "Event-Based Haptic Tapping with Grip Force Compensation," in Haptic Interfaces for Virtual Environment and Teleoperator Systems, 2006 14th Symposium on, 2006, pp. 117-123.

[23] E. C. Levi, "Complex-Curve Fitting," in IRE Trans. on Automatic Control. vol. AC-4, ed, 1959, pp. 37-44.

[24] M. J. D. Otis and B. J. Menelas, "Toward an augmented shoe for preventing falls related to physical conditions of the soil," in IEEE International Conference on Systems, Man, and Cybernetics (SMC), 2012, pp. 3281-3285.

[25] C. Antfolk, C. Balkenius, G. Lundborg, B. Rosén, and F. Sebelius, "Design and technical construction of a tactile display for sensory feedback in a hand prosthesis system," Biomedical engineering online, vol. 9 , p. 50, 2010.

[26] J. E. Dennis, Jr., and R.B. Schnabel, "Numerical Methods for Unconstrained Optimization and Nonlinear Equations," ed: PrenticeHall, 1983, pp. 221-228. 